\title{
Atrial Electromechanical Delay of Patients with COPD in Acute and Stable Periods
}

\author{
Gokhan Perincek $^{1, *}$ (D) Sema Avci ${ }^{2}$ and Ferdi Kahraman ${ }^{3}$ (iD) \\ ${ }^{1}$ MD, Kars Harakani State Hospital, Department of Pulmonology, Kars, Turkey \\ 2 MD, Amasya University Sabuncuoglu Serefeddin Research and Training Hospital, Department of Emergency Medicine, Amasya, Turkey \\ ${ }_{3}$ MD, Gebze Fatih State Hospital, Department of Cardiology, Kocaeli, Turkey
}

* Corresponding author: Gokhan Perincek, Kars Harakani State Hospital, Department of Pulmonology, Kars, Turkey. Tel: +905072504470;

Email: md.gokhanperincek@gmail.com

Received 2020 November 13; Revised 2020 December 25; Accepted 2021 January 03.

\begin{abstract}
Background: The increased level of the airway and systemic inflammation, worsened clinical symptoms, and impaired lung functions are regarded as the characteristics of chronic obstructive pulmonary disease (COPD). The COPD may cause right/left ventricle dysfunction, pulmonary hypertension, and cor pulmonale.

Objectives: The current study aimed to assess atrial electromechanical delay (AEMD) and echocardiographic changes and their relationship with inflammatory markers in subjects suffering from COPD during acute and stable periods.

Methods: This prospective study was carried out on a total of 45 patients (including 22 and 23 female and male participants, respectively) suffering from COPD exacerbation. The stable phase of the participants was considered the control group. The first and second echocardiography was conducted in the first $24 \mathrm{~h}$ and following 3 months, respectively. Conventional and Tissue Doppler Imaging was utilized for the evaluation of atrial conduction times and systolic-diastolic functions of the right-left heart. The inflammatory markers, including the c-reactive protein-to-albumin ratio (CAR), neutrophil-to-lymphocyte ratio (NLR), and platelet-to-lymphocyte ratio, were also measured in this study.

Results: In the stable period, there was a significant reduction in lateral/tricuspid, lateral/mitral, and septal AEMD. The evaluation of right ventricle basal, mid and vertical diameters, tricuspid annular plane systolic excursion, Amax tricuspid, tricuspid regurgitant velocity, systolic pulmonary arterial pressure, tricuspid annular systolic motion, left atrium diameter, left ventricle end-diastolic diameter, interventricular septum thickness, mitral early diastole/atrium systole ratio, systolic mitral motion, systolic septal motion, and heart rate showed significant differences after 3 months. In addition, there was a significant decrease in C-reactive protein, CAR, and NLR

Conclusion: In the stable period, a significant reduction was observed in AEMDs and there was an improvement in the systolic functions of the right-left heart. No correlation was observed between AEMDs and inflammatory markers.

Keywords: Atrial electromechanical delay, Chronic obstructive pulmonary disease, Echocardiography
\end{abstract}

\section{Background}

The progressive airflow limitation is the common characterization of chronic obstructive pulmonary disease (COPD). Furthermore, there is a direct association between COPD and an increased chronic inflammatory response in the airways (1). The exacerbation of COPD is defined by the increased level of the airway and systemic inflammation, worsened respiratory clinical symptoms, and impaired lung functions of patients (2). The COPD primarily affects the lungs; however, it has several important extrapulmonary effects, including cardiovascular system abnormalities contributing to the severity of the disease $(1,3)$.

The most important causes of mortality in patients with mild-to-moderate COPD are considered cardiovascular diseases, and cardiovascular involvement remains undiagnosed in many of these patients (4). The development, evaluation, and prevalence of cardiovascular comorbidities in COPD patients have not been totally clarified; nevertheless, clinically important manifestations, such as myocardial infarction, heart failure secondary to COPD, and arrhythmias, are the most common ones $(4,5)$. The COPD also affects the right ventricle (RV) as well as the left ventricle (LV) pulmonary blood vessels and causes pulmonary hypertension, cor pulmonale, and RV-LV dysfunction (6).

Atrial fibrillation (AF) as the most frequently encountered arrhythmia in clinical practice is related to increased mortality and morbidity (7). The COPD is independently associated with AF; nonetheless, the pathophysiological mechanism is not completely clarified (7). Increased atrial automaticity, triggered activity, micro reentry, and abnormal atrial tissue are the responsible mechanisms of $\mathrm{AF}(8)$.

Atrial electromechanical delay (AEMD) is the time interval between the beginning of the $P$ wave on the surface electrocardiogram (ECG) and starting point of the late diastolic wave (Am-wave) on Tissue Doppler Imaging (TDI) (9). The structural changes of atrial tissue cause delay between the electrical stimulation and mechanical contraction (9). Atrial tissue changes can cause prolongation of the $P$ wave on the surface ECG (10). The prolongation of the $\mathrm{P}$ wave can be 
observed in patients undergoing coronary artery bypass surgery and suffering from hypertrophic cardiomyopathy, right atrial dilatation, atrial septal defect, hypertension, and COPD due to affected atrial tissue (10). The COPD has an inflammatory process, and several studies have suggested the C-reactive protein-to-albumin ratio (CAR), neutrophil-tolymphocyte ratio (NLR), and platelet-to-lymphocyte ratio (PLR) as well-known biomarkers for the measurement of inflammation in patients with COPD (11-13).

\section{Objectives}

The current study aimed to assess AEMD and echocardiographic changes and their relationship with inflammatory markers in patients with COPD during acute and stable periods.

\section{Methods}

This prospective study was approved by the Ethics Committee of Medical Faculty of Kafkas University in Kars, Turkey, within February and July 2018. The present study was carried out on a total of 45 patients (including 22 and 23 female and male participants, respectively) suffering from COPD exacerbation, admitted to the Department of Emergency Medicine in Kars Harakani State Hospital with stages I-IV. The stable phase of the participants was regarded as the control group. The exacerbation period of COPD was considered to be an increase in any of the symptoms, including dyspnea, cough, sputum production, or sputum purulence.

Demographic profile, complete blood count, Creactive protein (CRP), CAR, NLR, PLR, pulmonary function tests, and transthoracic echocardiography were assessed in this study. Forced expiratory volume in the first second and forced vital capacity were calculated at the baseline using a spirometer (Spirolab III-MIR, Italy). Echocardiography was carried out for all the study subjects in the first $24 \mathrm{~h}$. After 3 months, the second echocardiography was performed by the same practitioner. All the patients were informed about the objectives of the study. In addition, written consent for all the procedures was obtained from the patients.

The exclusion criteria of the present study were patients with no previous COPD, valvular heart disease, wall-motion abnormality, uncontrolled hypertension, insulin-dependent diabetes mellitus, hypo/hyperthyroidism, anemia, renal failure, coronary artery disease, acute coronary syndrome, heart failure, structural heart diseases, atrioventricular conduction abnormalities, ejection fraction of $<50 \%$, pulmonary embolism, pneumonia, malignancy, or failure to comply with echocardiography, history of AF, and prior use of antiarrhythmic drugs. The diagnosis of COPD exacerbation was in line with the criteria established by the European Respiratory Society and American Thoracic Society $(14,15)$.

\subsection{Echocardiography}

Transthoracic echocardiography (EPIQ 7; Philips, USA) was evaluated by a practitioner according to a standard protocol in all the patients. The subjects were monitored using electrocardiographic leads and placed in the left lateral decubitus position. Echocardiographic images were obtained from the parasternal views (i.e., long axis and short axis), apical 4-chamber view, and subcostal view. Echocardiographic measurements were carried out at the end of expiration according to the suggestions of the American Society of Echocardiography/European Association of Echocardiography as follows (16):

1) measurement of the diameters of the RV in the apical view

2) measurement of the LV diameter and wall thickness in the parasternal view

3) measurement of the left atrial diameter in the parasternal view

4) measurement of aortic root diameters at the sinuses of Valsalva

5) measurement of the LV ejection fraction in the apical 4-chamber view by modified Simpson method

6) evaluation of the RV and LV functions as follows: a) maximal peak velocity of early diastolic flow (Emax), maximal peak velocity of atrial contraction (Amax), and ratio of these (Emax/Amax) calculated over the mitral and tricuspid valves; b) TDI calculated in the mitral and tricuspid lateral annulus at early diastole (Ea), atrium systole (Aa), and ratios of these $\mathrm{Ea} / \mathrm{Aa}$; c) ratio of Emax/Ea

7) aortic, tricuspid, mitral and pulmonary valvular assessment

8) tricuspid regurgitant velocity (TRV) registered by Continuous-wave Doppler

The measurement of AEMD was carried out using colored-TDI recordings. The AEMD was determined as the time interval between the beginning of echocardiographic $\mathrm{P}$ wave and starting point of Amwave (i.e., the late diastolic wave) in TDI registrations and measured from lateral/tricuspid, lateral/mitral, and septal annulus from apical 4-chamber views.

\subsection{Statistical Analysis}

Statistical analysis was carried out using SPSS software (version 23.0). The results of analyses were presented as mean \pm standard deviation and median (minimum-maximum) for the quantitative data. In addition, categorical variables were shown in percentage. Histogram and Kolmogorov-Smirnov test were used for the determination of the normal distribution. The paired samples t-test and Wilcoxon test were utilized for normally and non-normally distributed data in analyzing the changes with respect to dual time, respectively. A p-value of less than 0.05 was considered statistically significant. 


\section{Results}

Table 1 and Table 2 show the clinical features and inflammatory markers of the patients, respectively. The CRP, CAR, and NLR significantly decreased after 3 months $(\mathrm{P}<0.001)$. Table 3 tabulates the RV measurements, diastolic and systolic functions, and pulmonary artery pressure of the right heart in the patients. There were differences in RV basal, mid and vertical diameters, tricuspid annular plane systolic excursion (TAPSE), Amax tricuspid, TRV, systolic pulmonary arterial pressure (SPAP), and systolic motion tricuspid after 3 months $(\mathrm{P}<0.05)$.

Table 4 shows conventional and TDI parameters of the left heart-septum and atrial conduction times of the patients. After 3 months, there were differences in left atrium diameter, LV end-diastolic diameter, interventricular septum thickness, mitral Ea/Aa ratio, systolic mitral-septal motion, and heart rate $(\mathrm{P}<0.05)$. A significant reduction was observed in lateral/tricuspid, lateral/mitral, and septal AEMD among the patients $(\mathrm{P}<0.001)$.

The CAR did not correlate with lateral/tricuspid, lateral/mitral, and septal AEMD both on admission and following 3 months. There was no correlation between NLR and PLR with lateral/tricuspid, lateral/mitral, and septal AEMD both on admission and after 3 months. The CAR only negatively correlated with the tricuspid $\mathrm{Ea} / \mathrm{Aa}$ ratio on admission ( $\mathrm{r}=-0.316 ; \mathrm{P}=0.034)$. The CAR only positively correlated with TRV $(\mathrm{r}=0.322 ; \mathrm{P}=0.031)$. On admission, a correlation was observed between

\begin{tabular}{lc}
\hline Table 1. Clinical characteristics of patients & \\
\hline & $\begin{array}{c}\text { Median (min- } \\
\text { max)/n (\%) }\end{array}$ \\
\hline Age (year) & $69(44-86)$ \\
Gender & \\
Male & $23(51.1)$ \\
Female & $22(48.9)$ \\
Body mas index $\left(\mathbf{k g} / \mathbf{m}^{2}\right)$ & 27.43 \\
Forced expiratory volume in the first second & $17.51-43.27)$ \\
Forced vital capacity & $31(19-58)$ \\
Smoking status & $36(17-64)$ \\
Smoker & $5(11.1)$ \\
Ex-smoker & $20(44.4)$ \\
Never smoker & $20(44.4)$ \\
Comorbidities & \\
Hypertension & $22(48.9)$ \\
Diabetes mellitus & $4(8.9)$ \\
Hyperlipidemia & $1(2.2)$ \\
\hline
\end{tabular}

\begin{tabular}{lccc}
\hline Table 2. Inflammatory markers of patients & & & \\
\hline \multicolumn{1}{l}{ Acute period (min-max) } & Stable period (min-max) & P-value \\
\hline C-reactive protein (mg/L) & $1.15(0.13-47.47)$ & $0.6(0.02-6.18)$ & $<0.001$ \\
Albumin (g/L) & $4.3(3.26-4.78)$ & $4.46(3.65-5)$ & 0.009 \\
C-reactive protein-to-albumin ratio & $0.28(0.03-14.56)$ & $0.14(0-1.4)$ & $<0.001$ \\
Neutrophil (10^3/uL) & $5.44(2.51-14)$ & $5.14(2.37-7.98)$ & 0.032 \\
Lymphocyte $\left(\mathbf{1 0}^{\wedge} \mathbf{3} / \mathbf{u L}\right)$ & $1.56(0.03-10.7)$ & $1.69(1.03-3.88)$ & 0.033 \\
Neutrophil-to-lymphocyte ratio & $3.65(0.75-197.1)$ & $0.22(0.06-0.43)$ & $<0.001$ \\
Platelet (10^3/uL) & $227(67-403)$ & $211(117-412)$ & $\mathrm{NS}$ \\
Platelet-to-lymphocyte ratio & $0.71(0.51-1.62)$ & $0.74(0.53-1.29)$ & $\mathrm{NS}$ \\
\hline NS: Nonsignificant & & & \\
\end{tabular}

NS: Nonsignificant

\begin{tabular}{|c|c|c|c|}
\hline & Acute period (min-max) & Stable period (min-max) & P-value \\
\hline \multicolumn{4}{|l|}{ Right heart } \\
\hline \multicolumn{4}{|l|}{ Dimensions } \\
\hline \multicolumn{4}{|l|}{ Right ventricle (mm) } \\
\hline Basal & $37.2(14.1-45)$ & $36(27-44)$ & $<0.001$ \\
\hline Mid & $28(18-38)$ & $25(17.5-37)$ & $<0.001$ \\
\hline Vertical & $46(34-68)$ & $54(43-69.4)$ & $<0.001$ \\
\hline Tricuspid annular plane systolic excursion (mm) & $21(13-29)$ & $23(15-30)$ & 0.006 \\
\hline \multicolumn{4}{|l|}{ Ventricular function } \\
\hline \multicolumn{4}{|l|}{ Diastolic function } \\
\hline Emax tricuspid (cm/sec) & $50(32-104)$ & $48(26-78)$ & NS \\
\hline Amax tricuspid (cm/sec) & $68(36-118)$ & $61(38-116)$ & 0.032 \\
\hline Emax/Amax tricuspid $(\mathrm{cm} / \mathrm{sec})$ & $0.71(0.51-1.62)$ & $0.74(0.53-1.29)$ & NS \\
\hline Ea (tricuspid) $(\mathrm{cm} / \mathrm{sec})$ & $8.29(3-18.5)$ & $7.8(4.31-16)$ & NS \\
\hline Aa (tricuspid) (cm/sec) & $18(8-27.5)$ & $16.9(9.19-33.3)$ & NS \\
\hline Ea/Aa tricuspid & $0.48(0.18-1.32)$ & $0.46(0.17-1.1)$ & NS \\
\hline Emax/Ea & $5.71(3.13)$ & $6.08(2.86-12.8)$ & NS \\
\hline \multicolumn{4}{|l|}{ Assessment of pulmonary hypertension } \\
\hline Tricuspid regurgitan velocity (m/sec) & $3.03(2.17-3.85)$ & $2.82(2.02-3.85)$ & $<0.001$ \\
\hline Systolic pulmonary arterial pressure (mmHg) & 37 (19-59) & $33(16-72)$ & 0.002 \\
\hline Systolic motion tricuspid & $12.5(6.4-22.9)$ & $13.3(9.6-27)$ & 0.004 \\
\hline
\end{tabular}

NS: Nonsignificant

Ea: Early diastole; Aa: Atrium systole 
Table 4. Echocardiographic findings of the left heart, septum, and atrial conduction times of patients

\begin{tabular}{|c|c|c|c|}
\hline & Acute period (min-max) & Stable period (min-max) & P-value \\
\hline \multicolumn{4}{|l|}{ Left heart } \\
\hline \multicolumn{4}{|l|}{ Dimensions } \\
\hline \multicolumn{4}{|l|}{ Left atrium (parasternal long axis) } \\
\hline Diameter (mm) & $38(28.9-46)$ & $36(30-45)$ & 0.012 \\
\hline \multicolumn{4}{|l|}{ Left ventricle (parasternal long axis) } \\
\hline End-diastolic diameter (mm) & $44(35-58)$ & $46(40-60)$ & $<0.001$ \\
\hline End-sistolic diameter ( $\mathrm{mm})$ & $28(18-46)$ & $28(21-40)$ & NS \\
\hline \multicolumn{4}{|l|}{ Left ventricle wall thickness } \\
\hline Interventricular septum (mm) & $12(9-16.1)$ & $11.5(8.8-16.1)$ & $<0.001$ \\
\hline Posterior wall $(\mathrm{mm})$ & $10.2(8-16)$ & $10(8-12)$ & 0.001 \\
\hline \multicolumn{4}{|l|}{ Ventricular function } \\
\hline \multicolumn{4}{|l|}{ Systolic function } \\
\hline Left ventricular ejection fraction (\%) & $66.4(50-75)$ & $67(56-74)$ & NS \\
\hline \multicolumn{4}{|l|}{ Diastolic function } \\
\hline Emax mitral $(\mathrm{cm} / \mathrm{sec})$ & $63.4(39.8-121)$ & $66.5(38-130)$ & NS \\
\hline Amax mitral $(\mathrm{cm} / \mathrm{sec})$ & $86.8(64-149)$ & $86(63-136)$ & NS \\
\hline Emax/Amax mitral & $0.71(0.32-1.46)$ & $0.74(0.45-1.28)$ & NS \\
\hline Ea (tricuspid lateral mitral) $(\mathrm{cm} / \mathrm{sec})$ & $8.29(4.06-12.6)$ & $8.6(3.19-14.1)$ & NS \\
\hline Аа (tricuspid lateral mitral) $(\mathrm{cm} / \mathrm{sec})$ & $12.9(6-21.8)$ & $12(7.01-21)$ & NS \\
\hline $\mathrm{Ea} /$ Aa mitral & $0.64(0.25-1.77)$ & $0.7(0.25-1.4)$ & 0.010 \\
\hline Aortic root diameter $(\mathrm{cm})$ & $3.5(2.24-4.2)$ & $3.48(2.8-4.5)$ & NS \\
\hline \multicolumn{4}{|l|}{ Septum } \\
\hline Ea (tricuspid septal) (cm/sec) & $5.9(3.19-9.6)$ & $5.8(2.9-10.4)$ & NS \\
\hline $\mathrm{Aa}$ (tricuspid septal) (cm/sec) & $11.6(7.01-21)$ & $11.3(7.6-18.3)$ & NS \\
\hline Ea/Aa septal & $0.52(0.23-0.94)$ & $0.58(0.31-0.8)$ & NS \\
\hline Emax/Ea & $10.75(5.21-20.86)$ & $11.02(5.65-22.03)$ & NS \\
\hline Mitral systolic motion & $8.41(4.97-12.4)$ & $9.4(4.97-15.8)$ & 0.001 \\
\hline Septal systolic motion & $7.2(4-10.8)$ & $8(5.8-10.5)$ & 0.001 \\
\hline Heart rate (beats/min) & $96(72-132)$ & $86(56-108)$ & $<0.001$ \\
\hline \multicolumn{4}{|l|}{ Atrial electromechanical delay } \\
\hline Lateral/tricuspid (msec) & $40(14-84)$ & $25(11-67)$ & $<0.001$ \\
\hline Lateral/mitral (msec) & $70(42-102)$ & $56(35-88)$ & $<0.001$ \\
\hline Septal (msec) & $50(18-77)$ & $35(18-77)$ & $<0.001$ \\
\hline
\end{tabular}

NS: Nonsignificant

Ea: Early diastole; Aa: Atrium systole

lateral/mitral AEMD with weight and body mass index (BMI) ( $\mathrm{r}=0.347 ; \mathrm{P}=0.020 ; \mathrm{r}=0.418 ; \mathrm{P}=0.004$; respectively).

\section{Discussion}

There is an association between the exacerbation of COPD with increased systemic and airway inflammation. Furthermore, this inflammation is responsible for worsening clinical symptoms and diminishing lung functions (2). Several biochemical markers have been studied for the severity of the disease, inflammatory status, and morbidity and mortality of COPD. In the present study, CRP, CAR, and NLR we evaluated for inflammatory status and the serum levels of these biomarkers decreased after the exacerbation period. The decrease of these markers was an expected condition for the patients.

Based on the literature, NLR and CRP are wellknown biomarkers for inflammation in patients with COPD (2, 11-13). In addition to CRP, albumin is a negative acute phase reactant showing inflammatory response and CAR positively correlates with severe inflammation and infection, especially in critically ill patients $(12,17)$. The CAR is a more valuable biomarker than CRP or albumin alone in this regard (12).
In the current study, the RV basal and mid diameters were higher and the vertical diameter was lower in the exacerbation period. There was no significant change in the RV diastolic function except in Amax. In echocardiographic practice, RV has a crescent shape and this crescentic geometry is distinct from the cone-shaped $\operatorname{LV}(18,19)$. Pulmonary arterial hypertension (PAH) as a kind of pre-capillary pulmonary hypertension is associated with normal or low wedge pressure and increasing this pressure may cause RV morphological structure change (20). The increased pressure and wall stress in the RV cause fibrosis, systolic-diastolic dysfunction, and high oxygen consumption (20).

The increased end-diastolic pressure with higher RV mass brings about ischemia and RV failure (20). The COPD is one of the causes of PAH, and exacerbation may impair RV function (3). The increased intrathoracic pressure of COPD patients reduces RV output, ventricular filling, stroke volume, and cardiac output; nevertheless, dynamic changes in heart size throughout the respiration cycle have unknown etiology (21). Increased intrathoracic pressure may also change the RV diameters.

It was observed that tricuspid annular systolic motion and TAPSE of patients increased the exacerbation period. Systolic motion tricuspid 
indicates that RV systolic function and TAPSE are useful views of RV ejection fraction, which also demonstrate the RV systolic function $(22,23)$. After 3 months, systolic motion mitral-septal increased as well as tricuspid annular systolic motion. These findings showed that both RV and LV systolic functions improved. In COPD patients, the RV is prone to changes in pressure consisted of the remodeling of the lung (23). The decrease in these parameters indicates the improvement of systolic functions of the right heart at the end of 3 months.

The TRV and pulmonary artery pressure decreased after an exacerbation. The TRV correlates with SPAP and is calculated using the pressure gradient between the RV and right atrium (24). Tricuspid regurgitation is usually caused by increased RV afterload and pulmonary hypertension secondary to chronic lung diseases, such as COPD, responsible for increased regurgitation (25). In the present study, it was assumed that the diameters and systolic functions of the right heart were related to COPD severity; nonetheless, the diastolic functions of the right heart were unrelated to this clinical picture. In patients with exacerbation, the left atrial diameter and interventricular septum wall thickness were higher and the end-diastolic diameter was lower.

Tachycardia has been frequently observed during the exacerbation period of COPD and is a common dysrhythmia in COPD patients (26). A substantial decrease in diastole during tachycardia may result in the reduction of end-diastolic diameter. The LV cannot relax in the diastole due to tachycardia, and the LV end-diastolic diameter and septum thickness can increase.

The results of the present study showed that atrial conduction times decreased after the exacerbation and this period may cause the prolongation of AEMD. The prolongation of atrial conduction times and nonuniform extension of sinus impulses are the wellrecognized electrophysiological characteristics of the atrium prone to fibrillation (27). The COPD is independently associated with AF; however, welldefined pathophysiological mechanisms are not completely perceived (7). In COPD patients, right atrial dilatation, fibrosis, hypoxia, hypercapnia, oxidative stress, respiratory drugs (e.g., beta-agonist and anticholinergic agents), prolonged AEMD, and inflammation may be associated with $\operatorname{AF}(7,28)$.

In the present study, the inflammatory markers, including CAR, CRP, and NLR, were higher and atrial prolongation times were longer during exacerbation. After this period, inflammation and atrial conduction times decreased; nevertheless, these markers did not correlate with atrial conduction times (i.e., lateral/mitral, lateral/tricuspid, and septal). Lateral/ mitral AEMD correlated with weight and BMI after acute period. In patients with COPD, other factors may cause AEMD rather than inflammation.

\subsection{Limitations}

The current investigation was cross-sectional with a relatively small sample size. In the present study, the relationship between decreased inflammation and AEMD was not shown. It is required to carry out long-term follow-up and large-scale prospective studies for the determination of predictive factors of AEMD in COPD patients. More sensitive markers should be used for the assessment of inflammation. It is also required to perform a more comprehensive study comparing the risk factors of prolonged AEMD in COPD patients, such as hypoxia, hypercapnia, respiratory drugs, fibrosis, oxidative stress, atrial dilatation, and inflammation.

\section{Conclusion}

A significant reduction in AEMDs and improvement of systolic functions of the right-left heart were observed in the stable period. In addition, there was no correlation between AEMDs and inflammatory markers.

\section{Acknowledgments}

None declared.

\section{Footnotes}

Authors' Contribution: The authors stated that each author has contributed to, read, and approved the manuscript.

Conflict of Interests: The authors declare that there is no conflict of interest.

Ethical Approval: The current study was approved by the Medical Faculty of Kafkas University.

Funding/Support: The present study was not financially supported by any institutions.

Informed consent: Verbal and written informed consent was obtained from all the participants.

\section{References}

1. Rawy AM, Fathalla D. Left ventricular diastolic dysfunction in patients with chronic obstructive pulmonary disease (COPD), prevalence and association with disease severity: using tissue Doppler study. Egypt J Chest Dis Tuberc. 2015;64(4):785-92. doi: 10.1016/j.ejcdt.2015.06.010.

2. Yao CY, Liu XL, Tang Z. Prognostic role of neutrophillymphocyte ratio and platelet-lymphocyte ratio for hospital mortality in patients with AECOPD. Int J Chron Obstruct Pulmon Dis. 2017;12:2285-90. doi: 10.2147/COPD.S141760. [PubMed: 28814856].

3. Ozben B, Eryuksel E, Tanrıkulu AM, Papila N, Ozyigit T, Celikel $\mathrm{T}$, et al. Acute Exacerbation Impairs Right Ventricular Function in COPD Patients. Hellenic J Cardiol. 2015;56(4):324-31. [PubMed: 26233773].

4. Patel AR, Hurst JR. Extrapulmonary comorbidities in chronic obstructive pulmonary disease: state of the art. Expert Rev Respir Med. 2011;5(5):647-62. doi: 10.1586/ers.11.62. [PubMed: 21955235]

5. Ferixa X, Portillo K, Pare C, Garcia-Aymerich J, Gomez FP, Benet $M$, et al. Echocardiographic abnormalities in patients with 
COPD at their first hospital admission. Eur Respir J. 2013; 41(4):784-9. doi: 10.1183/09031936.00222511. [PubMed: 23018914].

6. Gupta NK, Agrawal RK, Srivastav AB, Ved ML. Echocardiographic evaluation of heart in chronic obstructive pulmonary disease patient and its co-relation with the severity of disease. Lung India. 2011;28(2):105-9. doi: 10.4103/09702113.80321. [PubMed: 21712919].

7. Goudis CA. Chronic obstructive pulmonary disease and atrial fibrillation: an unknown relationship. J Cardiol. 2017; 69(5):699-705. doi: 10.1016/j.jjcc.2016.12.013. [PubMed: 28188041].

8. Markides V, Schilling RJ. Atrial fibrillation: classification, pathophysiology, mechanisms and drug treatment. Heart. 2003;89(8):939-43. doi: 10.1136/heart.89.8.939. [PubMed: 12860883].

9. Ari H, Ari S, Akkaya M, Aydın C, Emlek N, Sarıgul OY, et al. Predictive value of atrial electromechanical delay for atrial fibrillation recurrence. Cardiol J. 2013;20(6):639-47. doi: 10.5603/CJ.2013.0164. [PubMed: 24338542].

10. Caglar IM, Dasli T, Caglar FN, Teber MK, Ugurlucan M, Ozmen G. Evaluation of atrial conduction features with tissue Doppler imaging in patients with chronic obstructive pulmonary disease. Clin Res Cardiol. 2012;101(8):599-606. doi: 10.1007/s00392-012-0431-7. [PubMed: 22391986].

11. El-Gazzar AG, Kamel MH, Elbahnasy OKM, El-Naggar ME. Prognostic value of platelet and neutrophil to lymphocyte ratio in COPD patients. Expert Rev Respir Med. 2020;14(1):111-6. doi: 10.1080/17476348.2019.1675517. [PubMed: 315779119].

12. Akkececi NS, Cetin GY, Gogebakan H, Acipayam C. The Creactive protein/albumin ratio and complete blood count parameters as indicators of disease activity in patients with Takayasu arteritis. Med Sci Monit. 2019;25:1401-9. doi: 10.12659/MSM.912495. [PubMed: 30792377].

13. Günay E, Sarınç Ulaşlı S, Akar O, Ahsen A, Günay S, Koyuncu T, et al. Neutrophil-to-lymphocyte ratio in chronic obstructive pulmonary disease: a retrospective study. Inflammation. 2014;37(2):374-80. doi: 10.1007/s10753-013-9749-1. [PubMed: 24078279].

14. Burge S, Wedzicha JA. COPD exacerbations: definitions and classifications. Eur Respir J. 2003;41:46s-53s. doi: 10.1183/ 09031936.03.00078002. [PubMed: 12795331].

15. COPD. ATS Official Documents. Available at: URL: https://www.thoracic.org/statements/copd.php; 2018.

16. Lang RM, Bierig M, Devereux RB, Flachskampf FA, Foster E, Pelikka PA, et al. Recommendations for chamber quantification: a report from the American society of echocardiography's guidelines and standards committee and the chamber quantification writing group, developed in conjunction with the European association of echocardiography, a branch of the European society of cardiology. J Am Soc Echocardiogr. 2005;18(12):1440-63. doi: 10.1016/j.echo.2005.10.005. [PubMed: 16376782].

17. Atalay E, Erdogdu HI, Tur BK, Deniz Balyen LS, Karabag Y, Ardıc
S. The relationship between $\mathrm{C}$ reactive protein /albumin ratio and 1-year mortality in hospitalized elderly COPD patients with acute exacerbation. Turk J Geriatr. 2019;22(1):9-17.

18. Ho SY, Nihoyannopoulos P. Anatomy, echocardiography, and normal right ventricular dimensions. Heart. 2006;92(Suppl 1):2-13. doi: 10.1136/hrt.2005.077875. [PubMed: 6543598].

19. Ryan JJ, Archer SL. The right ventricle in pulmonary arterial hypertension: disorders of metabolism, angiogenesis and adrenergic signaling in right ventricular failure. Circ Res. 2014;115(1):176-88. doi: 10.1161/CIRCRESAHA.113.301129. [PubMed: 24951766].

20. Foschi M, Di Mauro M, Tancredi F, Capparuccia C, Petroni R, Leonzio $\mathrm{L}$, et al. The dark side of the moon: the right ventricle. $J$ Cardiovasc Dev Dis. 2017;4(4):18. doi: 10.3390/jcdd4040018. [PubMed: 29367547].

21. Xu Y, Yamashiro T, Moriya H, Tsubakimoto M, Tsuchiya N, Nagatani $Y$. Hyperinflated lungs compress the heart during expiration in COPD patients: a new finding on dynamicventilation computed tomography. Int I Chron Obstruct Pulmon Dis. 2017;12:3123-31. doi: 10.2147/COPD.S145599. [PubMed: 29123390].

22. Meluzín J, Spinarová L, Bakala J, Toman J, Krejcí J, Hude P, et al. Pulsed Doppler tissue imaging of the velocity of tricuspid annular systolic motion; a new, rapid, and non-invasive method of evaluating right ventricular systolic function. Eur Heart J. 2001;22(4):340-8. doi: 10.1053/euhj.2000.2296. [PubMed: 11161953].

23. Terzano C, Romani S, Gaudio C, Pelliccia F, Serao M, Vitarelli A. Right heart functional changes in the acute, hypercapnic exacerbations of COPD. Biomed Res Int. 2014;2014:596051. doi: 10.1155/2014/596051. [PubMed: 25050365].

24. Bossone E, Rubenfire M, Bach DS, Ricciardi M, Armstrong WF. Range of tricuspid regurgitation velocity at rest and during exercise in normal adult men: implications for the diagnosis of pulmonary hypertension. J Am Coll Cardiol. 1999;33(6):1662-6. doi: 10.1016/s0735-1097(99)00055-8. [PubMed: 10334439].

25. Badano LP, Muraru D, Enriquez-Sarano M. Assessment of functional tricuspid regurgitation. Eur Heart J. 2013;34(25): 1875-84. doi: 10.1093/eurheartj/ehs474. [PubMed: 23303656].

26. Falk JA, Kadiev S, Criner GJ, Scharf SM, Minai OA, Diaz P. Cardiac disease in chronic obstructive pulmonary disease. Proc Am Thorac Soc. 2008;5(4):543-8. doi: 10.1513/pats.200708142ET. [PubMed: 18453369].

27. Yagmur J, Yetkin O, Cansel M, Acıkgoz N, Ermis N, Karakus Y, et al. Assessment of atrial electromechanical delay and influential factors in patients with obstructive sleep apnea. Sleep Breath. 2012;16(1):83-8. doi: 10.1007/s11325-010-0477-6. [PubMed: 21221821].

28. Acar G, Kahraman H, Akkoyun M, Kilinc M, Zencir C, Yusufoglu E, et al. Evaluation of atrial electromechanical delay and its relationship to inflammation and oxidative stress in patients with chronic obstructive pulmonary disease. Echocardiography. 2014;31(5):579-85. doi: 10.1111/echo.12442. [PubMed: 24372655] 